\title{
Cocoa diet diminishes the anti-collagen humoral response in collagen-induced arthritis in rats
}

\author{
S Ramos-Romero, T Pérez-Berezo, F J Pérez-Cano, C Castellote, M Castell, A Franch ${ }^{*}$ \\ From 5th European Workshop on Immune-Mediated Inflammatory Diseases \\ Sitges-Barcelona, Spain. 1-3 December 2010
}

\section{Introduction}

Collagen type II-induced arthritis (CIA) shares immunological and pathological characteristics with human rheumatoid arthritis. Anti-collagen (CII) autoantibodies appear to be a primary mechanism of immunopathogenesis in this model [1]. On the other hand, previous studies have shown that rats fed cocoa exhibit a lower antibody production after ovoalbumin immunization [2].

\section{Aim}

The objective of the present study was to ascertain the effect of a high cocoa diet on humoral response produced in rats with CIA.

\section{Methods}

CIA was induced in female LOU rats fed with a $10 \%$ cocoa diet or standard chow. Blood samples were collected at days 14, 21 and 27 post-induction. Concentration of serum anti-CII Ig, IgM, IgG1, IgG2a, IgG2b and IgG2c antibodies were determined by ELISA. At day 27 post-induction, inguinal lymph nodes (ILN) were excised and cells were isolated. Anti-CII IgM- and IgG-secreting cells from ILN were enumerated by ELISPOT technique.

\section{Results}

Cocoa diet reduced total serum anti-CII antibody concentration 4 weeks postinduction $(\mathrm{P}<0.01)$. Anti-CII IgM antibodies were reduced by cocoa diet only in the early phase $(\mathrm{P}<0.05$ at day 14 post-induction). However, antiCII IgG antibodies were differentially influenced by cocoa intake and the concentration of those antibodies belonging to IgG2a and IgG2c isotypes was diminished by cocoa diet, mainly in the late arthritic phase $(\mathrm{P}<0.05$ at day 27 post-induction). Anti-CII IgG2b concentration was not significantly affected by cocoa intake during the

Dept. of Physiology, Faculty of Pharmacy, University of Barcelona, Spain studied period. Anti-CII IgG-secreting cell number was not significantly modified by cocoa diet, but anti-CII IgM-secreting cell counts were significantly diminished in ILN from CIA rats fed cocoa $(\mathrm{P}<0.05)$.

\section{Conclusion}

Cocoa diet reduced the synthesis of anti-CII autoantibodies in the collagen-induced arthritis model.

Published: 25 November 2010

\section{References}

1. Holmdahl R, Lorentzen JC, Lu S, Olofsson P, Wester L, Holmberg J, Pettersson $U$ : Arthritis induced in rats with non-immunogenic adjuvants as models for rheumatoid arthritis. Immunol Rev 2001, 184:184-202.

2. Pérez-Berezo T, Ramiro-Puig E, Pérez-Cano FJ, Castellote C, Permanyer J, Franch A, Castell M: Influence of a cocoa-enriched diet on specific immune response in ovalbumin-sensitized rats. Mol Nutr Food Res 2009, 53:389-397.

doi:10.1186/1479-5876-8-S1-P25

Cite this article as: Ramos-Romero et al:: Cocoa diet diminishes the anticollagen humoral response in collagen-induced arthritis in rats. Journal of Translational Medicine 2010 8(Suppl 1):P25. 